\title{
Observations of the brightest UHE Gamma-Ray Sources With the LHAASO-KM2A
}

\section{Sha Wu, ${ }^{1, *}$ SongZhan Chen ${ }^{1}$ and Ruo-Yu Liu ${ }^{2}$ on behalf of the LHAASO Collaboration}

(a complete list of authors can be found at the end of the proceedings)

\author{
${ }^{1}$ Key Laboratory of Particle Astrophyics \& Experimental Physics Division \& Computing Center, Institute \\ of High Energy Physics, Chinese Academy of Sciences, 100049 Beijing, China \\ ${ }^{2}$ School of Astronomy and Space Science, Nanjing University, 210023 Nanjing, Jiangsu, China \\ E-mail: wusha@ihep.ac.cn
}

\begin{abstract}
Cosmic rays are high-energy charged particles that come from outer space. They play an important role in the evolution of our Galaxy. Gamma-ray emission produced by cosmic rays is a direct probe of cosmic rays and their accelerators. As a key sub-array of the Large High Altitude Air Shower Observatory (LHAASO), KM2A is the most sensitive gamma-ray detector at ultra-high energy (UHE, >100 TeV) band. Here, we report four of the brightest UHE sources measured by LHAASO, LHAASO J1825-1326, LHAASO J1908+0621, LHAASO J2108+5157 and LHAASO J2226+6057. The morphology and spectral energy distribution of these sources are studied respectively using the KM2A data collected from December 2019 to December 2020. The origin of the UHE gamma-ray emission is also discussed taking into account the implications from multi-wavelength observations.
\end{abstract}

$37^{\text {th }}$ International Cosmic Ray Conference (ICRC 2021)

July 12th - 23rd, 2021

Online - Berlin, Germany

\footnotetext{
*Presenter
} 


\section{Introduction}

Galactic cosmic rays (CRs) reach energies of at least a few petaelectronvolts $\left(\mathrm{PeV}, 10^{15} \mathrm{eV}\right)$, the knee in the particle spectrum. This implies that our Galaxy contains PeV accelerators (PeVatrons). Identification of the PeVatrons is a prime objective towards understanding of the origin of CRs. The possible candidates include supernova remnants (SNRs) [1], Galactic Center [2], young massive star clusters [3] and so on. The typical energy of gamma rays produced in the interaction of CRs with ambient medium is about $10 \%$ of the parent CR energy. A decisive indication of acceleration of $\mathrm{PeV}$ protons is the observations of ultra-high energy (UHE; $\mathrm{E} \geq 0.1 \mathrm{PeV}$ ) gamma-rays.

The Large High Altitude Air Shower Observatory (LHAASO) is a new-generation complex EAS array being built at $4410 \mathrm{~m}$ a.s.l. near Daocheng, in the Sichuan province of China [4]. With large field of view and high duty cycle, LHAASO can survey a large fraction of the sky in the range of declination from $-15^{\circ}$ to $75^{\circ}$. The expected sensitivity of LHAASO above $30 \mathrm{TeV}$ is at least 10 times higher than current experiments. Therefore, LHAASO is a suitable tool to detect and study PeVatrons within our Galaxy. Recently, LHAASO reported the detection of 12 UHE gamma-ray sources with a statistical significance greater than seven standard deviations [5]. The photons detected by LHAASO far beyond $100 \mathrm{TeV}$ prove the existence of Galactic PeVatrons. It is likely that the Milky Way is full of these particle accelerators.

This paper mainly introduces three of the UHE gamma-ray sources detected by LHAASO as reported in [5] and another one reported in [6]. It is organized as following. In Section 2, the design and performance of LHAASO detectors are introduced. Section 3 describes the analysis method. In section 4, we report the results of the three sources.

\section{The LHAASO Detectors Array}

LHAASO mainly aims to very high energy gamma-ray astronomy and cosmic rays with energies in $10^{11}-10^{18} \mathrm{eV}$ [7]. It consists of three sub-arrays: the Square Kilometre Array (KM2A), the Water Cherenkov Detector Array (WCDA), and the Wide-Field Air Cherenkov Telescope Array (WFCTA). As the major array of LHAASO, KM2A contains 5195 electromagnetic particle detectors (EDs) and 1188 muon detectors (MDs), covering an area of $1.3 \mathrm{~km}^{2}$. Each ED consists of 4 plastic scintillation tiles covered by a $0.5-\mathrm{cm}$-thick lead plate to convert the gamma rays to electron-positron pairs and improve the angular resolution of the array. The EDs detect the electromagnetic particles in the shower, which are used to reconstruct the primary direction, core location and energy. Each MD includes a cylindrical water tank with a diameter of $6.8 \mathrm{~m}$ and a height of $1.2 \mathrm{~m}$. The tank is buried under $2.5 \mathrm{~m}$ of soil to shield against the high energy electrons/positrons and photons of the showers. The MDs are used to detect the muon component of showers, which is used to discriminate between gamma-ray and hadron induced showers.

Half of the KM2A array including 2365 EDs and 578 MDs has been put into operation since December 2019. According to the observation of Crab Nebula, the detector performance has been tested [8]. The core resolution ranges from $2-4 \mathrm{~m}$ and the angular resolution ranges from $0.2^{\circ}-0.3^{\circ}$ for events at $100 \mathrm{TeV}$. The energy resolution is about $24 \%$ at $20 \mathrm{TeV}$ and $13 \%$ at $100 \mathrm{TeV}$, for showers with zenith angle less than $20^{\circ}$. The KM2A half-array data set, collected from 27th December 2019 to 24th November 2020, were used in this analysis. 


\section{Analysis Methods}

The pipeline of KM2A data analysis presented in [8] is designed for surveying the whole sky in the range of declination from $-15^{\circ}$ to $75^{\circ}$. The same analysis methods are directly adopted in this work. The significances of sources are computed using a likelihood analysis given a specific source model. The likelihood ratio test statistic $(T S)$ is defined as:

$$
T S=2 \ln \frac{L_{S+b}}{L_{b}}
$$

where, $L_{s+b}$ is the maximum likelihood of the alternative hypothesis ( source model + background) and $L_{b}$ is the maximum likelihood of the null hypothesis (background only). The spectral energy distributions (SED) of sources are estimated with the forward-folding method described in [8]. The SED of this source is assumed to follow a power-law spectrum $d N / d E=\phi_{0}(E / 20 \mathrm{TeV})^{-\alpha}$. The best-fit values of $\phi_{0}$ and $\alpha$ are obtained by the least-squares fitting method.

\section{Results}

In the following, the results of the significance maps and spectral fits for the four sources are reported, respectively. The spectra are fitted with a log-parabola (LOG) function in the form of $[E /(10 \mathrm{TeV})]^{-a-b l o g}[E /(10 \mathrm{TeV})]$ and a power-law function of $E^{-\Gamma}$.

\subsection{LHAASO J1825-1326}

LHAASO J1825-1326 (Figure. 1) is detected at an $18.0 \sigma$ level above $25 \mathrm{TeV}$ with an extension of $\sigma=0.30^{\circ} \pm 0.06^{\circ}$. The source has a spectrum that is fit by a log-parabola with $a=0.92, b=1.19$ $\left(A I C_{L O G}=11.6\right)$ and a power-law with $\Gamma=3.36\left(A I C_{P L}=14.8\right)$. There are two energetic pulsars, PSR J1826-1334 and PSR J1826-1256, located in the source region, which may be the energy sources of the UHE emission. In the VHE gamma-ray regime, HESS detected an energy dependent morphology which is consistent with the leptonic origin of the gamma-ray emission[9]. In the future, We will dedicate an analysis to LHAASO J1825-1326 and try to figure out the physical origin. 

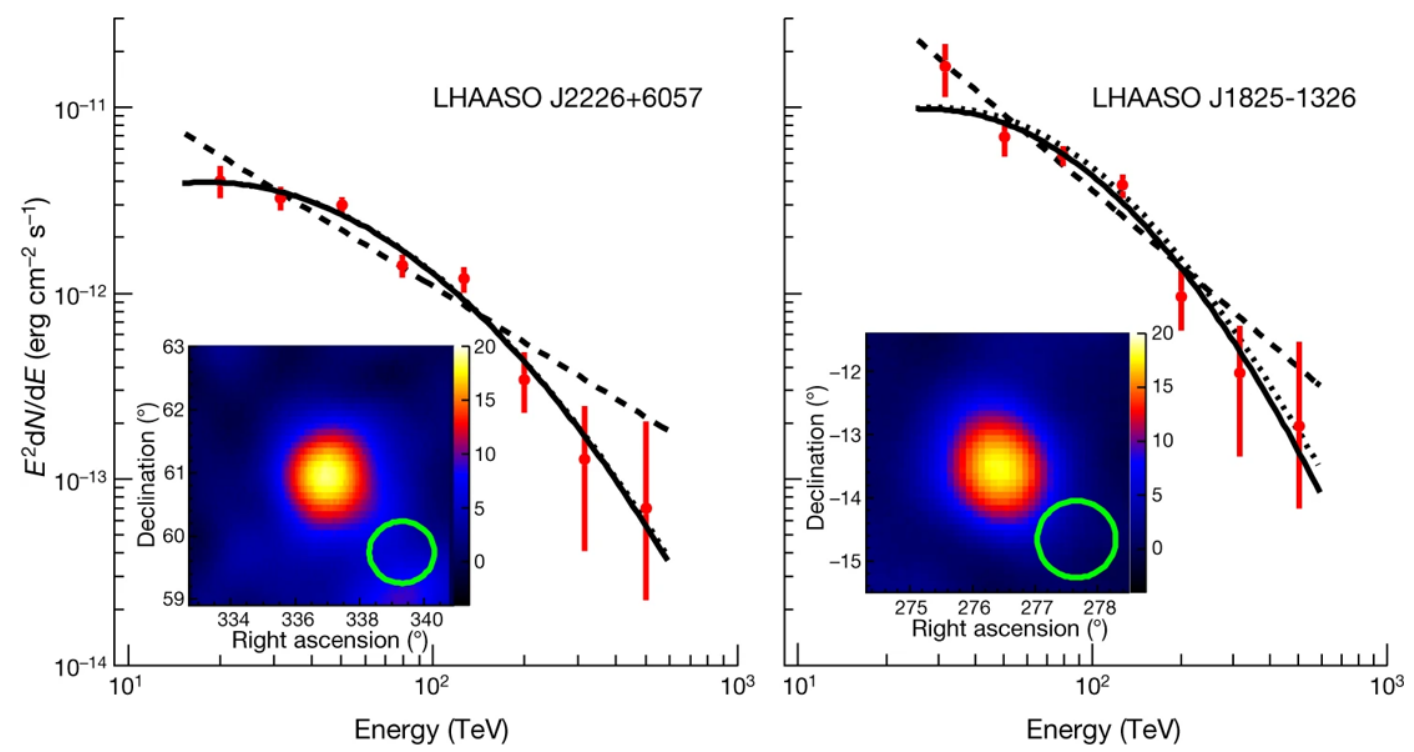

Figure 1: Spectral energy distributions and significance maps of LHAASO J2226+6057 and LHAASO J1825-1326 [5].

\subsection{LHAASO J1908+0621}

LHAASO J1908+0621 is consistent with The MGRO J1908+06 which was discovered by the MILAGRO collaboration [10] and later confirmed with the HESS atmospheric Cherenkov telescope [11]. It spatially associated with a middle-aged supernova remnant (SNR) G40.5-0.5 [12] and an energetic gamma-ray pulsar PSR J1907+0602 [13]. The age of G40.05-0.5 is estimated between 20 and $40 \mathrm{kyr}$ and the distance is uncertain. The PSR J1907+0602 has a characteristic age of $19.5 \mathrm{kyr}$ and a spin-down luminosity of $\sim 3 \times 10^{36} \mathrm{ergs}^{-1}$. The distance of PSR J1907+0602 was estimated to be $3.2 \mathrm{kpc}$. The nature of MGRO J1908+06 remains unrevealed and a single accelerator cannot explain the whole set of multiwavelength data.

The significance map around LHAASO J1908+0621 with $E_{r e c} \geq 25 \mathrm{TeV}$ are shown in Figure 2. We use the two-dimensional Gaussian model template to study the morphology of the source. The centroid of gamma-ray emissions with energies above $25 \mathrm{TeV}$ is R.A. $=287.04^{\circ} \pm 0.03_{\text {stat }}^{\circ}$, Dec. $=6.24^{\circ} \pm 0.04_{\text {stat }}^{\circ}(\mathrm{J} 2000)$ and the extension is $\sigma=0.57^{\circ} \pm 0.03^{\circ}$. Taking into account the Gaussian extension of $0.57^{\circ}$, the resulting differential flux can be fitted by a log-parabola for $a=2.27, b=0.46$ and a power-law for $\Gamma=2.89$. The Akaike Information Criterion (AIC) of log-parabola and power-law are 15.1 and 30.1 .

The gamma-ray spectral points with energies from gigaelectronvolt to several hundred teraelectronvolts could be explained by accelerated electrons following a spectrum of $N(E) \propto$ $N_{e}^{-1.75} \exp \left\{-E_{e} /(800 \mathrm{TeV})\right\}$. Alternatively, the gamma-ray emission could be produced by protons accelerated up to PeV colliding with the ambient dense gas. The spectrum of accelerated protons is more complex which is a broken power law with an exponential cutoff, with indices 1.2 and 2.7 below and above $25 \mathrm{TeV}$ and a cutoff energy of $1.3 \mathrm{PeV}$. 


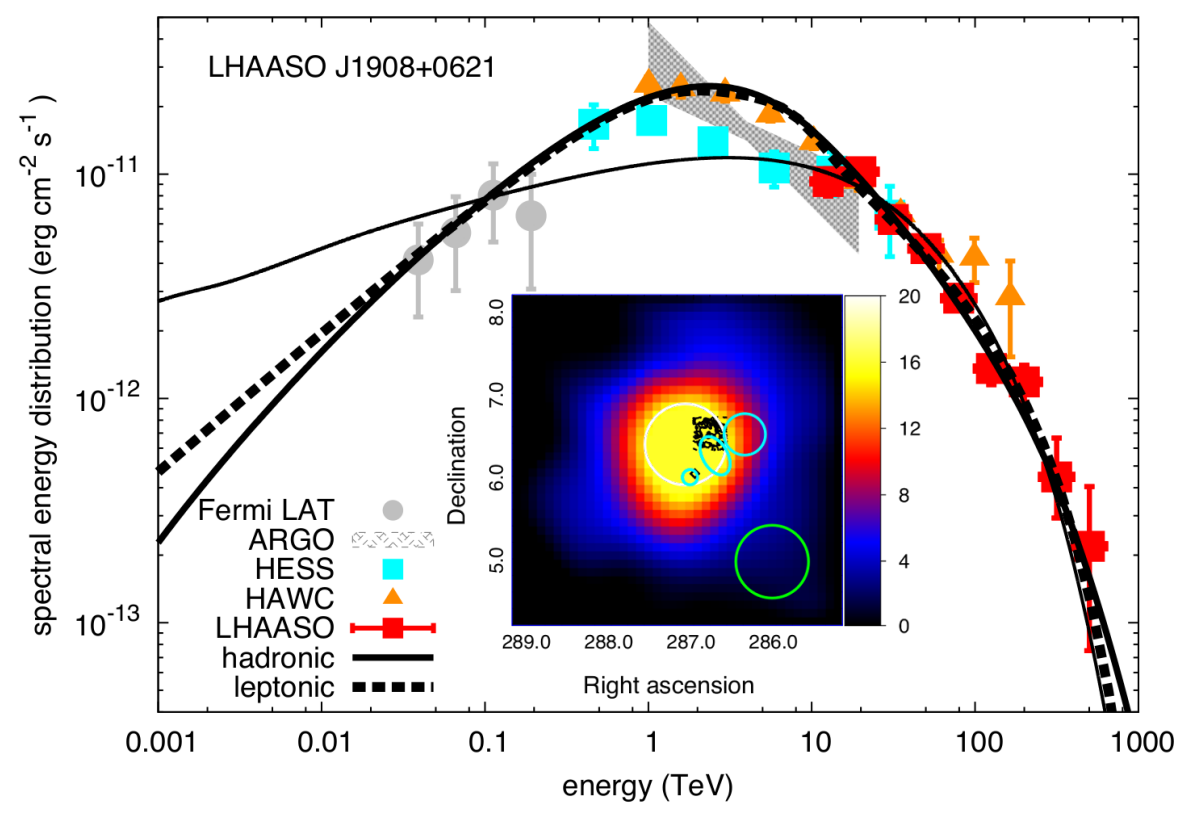

Figure 2: Phenomenological fits to the gamma-ray observations of LHAASO J1908+0621, and previous observations of potential counterparts [5].

\subsection{LHAASO J2108+5157}

LHAASO J2108+5157 is firstly discovered by LHAASO at approximately $9.5 \sigma$ and $8.5 \sigma$ significance level in both energy bands of $25-100 \mathrm{TeV}$ and $>100 \mathrm{TeV}$, respectively [6]. This source is not significantly favored as an extensive source with the angular extension smaller than the pointspread function of KM2A. An upper limit on the extension of the source is calculated to be $0.26^{\circ}$ at $95 \%$ confidence level (CL). Its spectrum from $20 \mathrm{TeV}$ to $500 \mathrm{TeV}$ is characterized by a power-law with index $-2.83 \pm 0.18_{\text {stat }}$. No obvious counterparts were found in the region of LHAASO J2108+5157 at other wavelengths. It is correlated with the molecular cloud [MML2017]4607. The UHE gammarays could be explained by interactions of protons with the ambient gas through the production and decay of $\pi^{0}$ mesons (As shown in Figure 3). The energy spectrum of protons follows a power-law with an exponential cutoff: $N(E) \propto N_{p}^{-2} \exp \left\{-E_{p} /(600 \mathrm{TeV})\right\}$. Other possible scenarios, such as a PWN, can also be invoked to explain the KM2A observed gamma-rays. The primary electron spectrum follows a power-law with an exponential cutoff: $N(E) \propto N_{e}^{-2.2} \exp \left\{-E_{e} /(200 \mathrm{TeV})\right\}$. Because of the absence of pulsar counterpart, the PWN scenario remains uncertain. So far, no conclusion about the origin of its UHE emission can be achieved. 


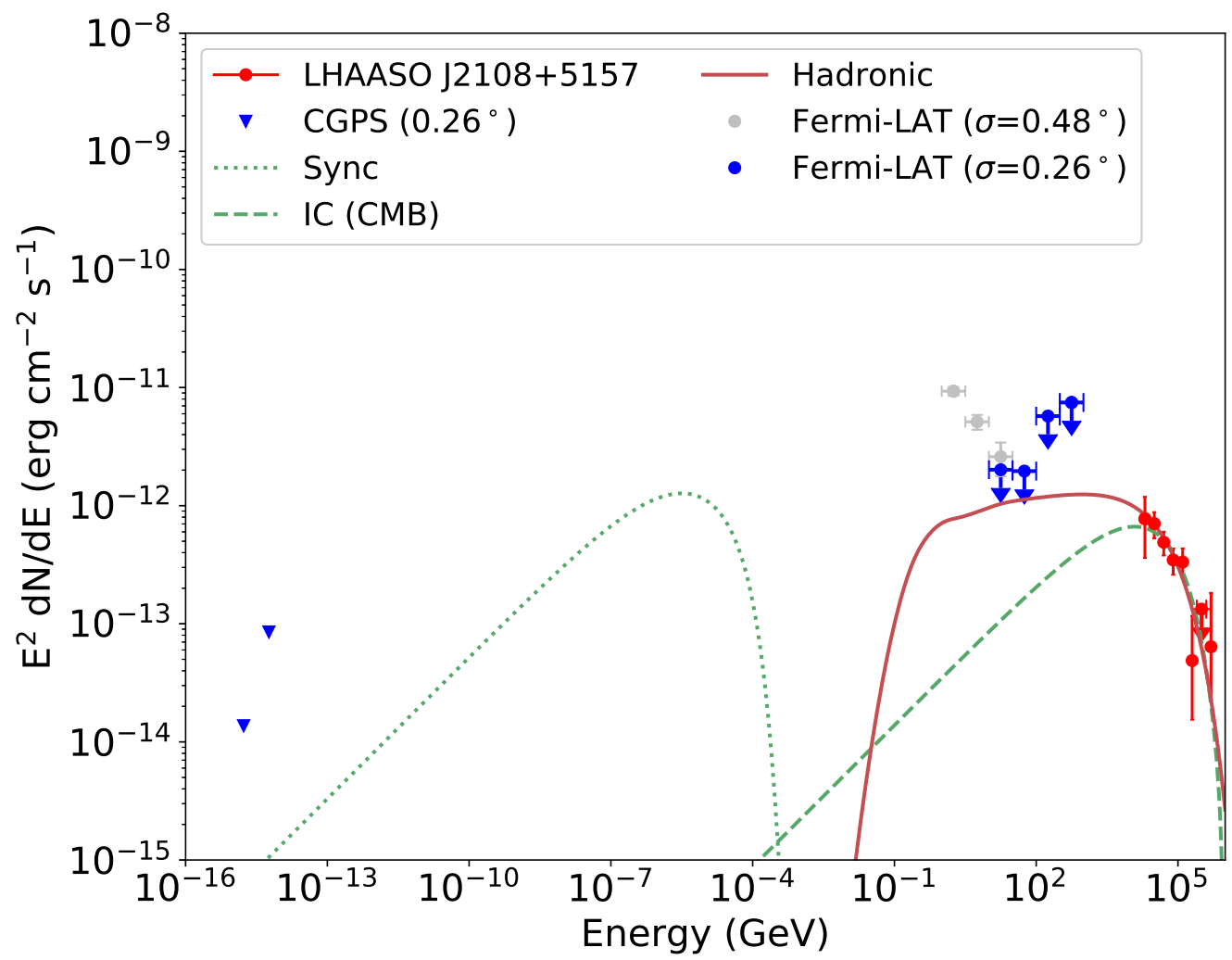

Figure 3: The multiwavelength SEDs of LHAASO J2108+5157 with hadronic and leptonic modeling [6]. The red points and arrows are the LHAASO-KM2A observations. The blue triangles are the radio fluxes. The grey points and blue arrows are the Fermi-LAT spectral points and upper limits.

\subsection{LHAASO J2226+6057}

LHAASO J2226+6057 (Figure. 1) is detected at an $18.0 \sigma$ level above $25 \mathrm{TeV}$, exhibiting a $\log$-parabola spectral for $a=1.56, b=0.88\left(A I C_{L O G}=12.3\right)$ and a power-law for $\Gamma=3.01$ $\left(A I C_{P L}=24.4\right)$. The detected highest photon energies is $0.57 \pm 0.19 \mathrm{PeV}$. The Source is spatially associated with SNR G106.3+2.7 which is a comet-shaped radio source, with a brighter "head" and an extended "tail" region. The "head" region also contains an off-center PWN in the north named "Boomerang" powered by the pulsar PSR J2229+6114, whose characteristic age and luminosity are $10 \mathrm{kyr}$ and $2.2 \times 10^{37} \mathrm{ergs}^{-1}$. The very-high-energy gamma-ray emission above $10 \mathrm{TeV}$ observed by AS $\gamma$ is well correlated with a molecular cloud rather than with the pulsar PSR J2229+6114 [14]. In the future, We will dedicate an analysis to LHAASO J2226+6057 from $100 \mathrm{GeV}$ to $1 \mathrm{PeV}$. This will provide crucial information to disentangle the origin of the gamma ray emission observed.

\section{Conclusions}

LHAASO has observed 12 UHE gamma-ray sources. It will play an important role in the identification of PeVatrons. Some simple discussion on the three most significantly detected sources are made. We will make a deep analysis of these three sources in the future. A new UHE gamma-ray source, LHAASO J2108+5157, is observed at an $8.5 \sigma$ level above $100 \mathrm{TeV}$. No obvious 
counterparts have been found, deeper multiwavelength observations will help to shed new light on this intriguing UHE source.

\section{References}

[1] Bell, A. R. 2013, Astroparticle Physics, 43, 56

[2] HESS Collaboration, Abramowski, A., Aharonian, F., et al. 2016, Nature Astronomy, 531, 476

[3] Aharonian, F., Yang, R., \& de Oña Wilhelmi, E. 2019, Nature Astronomy, 3, 561

[4] Cao, Z. 2010, Chin. Phys. C, 34, 249

[5] Cao, Zhen, F. A., An, Q., Axikegu, et al. 2021, Nature, doi:https://doi.org/10.1038/s41586021-03498-z

[6] The LHAASO collaboration. 2021, arXiv e-prints, arXiv:2106.09865

[7] He, H. 2018, Radiation Detection Technology and Methods, 2, 7

[8] Aharonian, F., An, Q., Axikegu, et al. 2020, Chin. Phys. C

[9] Liu, R.-Y., \& Yan, H. 2020, MNRAS, 494, 2618

[10] Abdo, A. A., Allen, B., Berley, D., et al. 2007, APJL, 664, L91

[11] Aharonian, F., Akhperjanian, A. G., Anton, G., et al. 2009, A \&A, 499, 723

[12] Downes, A. J. B., Pauls, T., \& Salter, C. J. 1980, A \&A, 92, 47

[13] Abdo, A. A., Ackermann, M., Ajello, M., et al. 2010, APJ, 711, 64

[14] Tibet AS $\gamma$ Collaboration, Amenomori, M., Bao, Y. W., et al. 2021, Nature Astronomy, 5, 460 


\section{Full Authors List: LHAASO Collaboration}

Zhen $\mathrm{Cao}^{1,2,3}$, F. Aharonian ${ }^{4,5}$, Q. An ${ }^{6,7}$, Axikegu ${ }^{8}$, L.X. Bai ${ }^{9}$, Y.X. Bai ${ }^{1,3}$, L.X. Bai ${ }^{9}$, Y.X. Bai ${ }^{1,3}$, Y.W. Bao ${ }^{10}$, D. Bastieri ${ }^{11}$, X.J. Bi ${ }^{1,2,3}$, Y.J. Bi ${ }^{1,3}$, H. Cai ${ }^{12}$, J.T. Cai ${ }^{11}$, Zhe Cao ${ }^{6,7}$, J. Chang ${ }^{13}$, J.F. Chang ${ }^{1,3,6}$, B.M. Chen ${ }^{14}$, E.S. Chen ${ }^{1,2,3}$, J. Chen ${ }^{9}$, Liang Chen $^{1,2,3}$, Liang Chen ${ }^{15}$, Long Chen ${ }^{8}$, M.J. Chen ${ }^{1,3}$, M.L. Chen ${ }^{1,3,6}$, Q.H. Chen $^{8}$, S.H. Chen ${ }^{1,2,3}$, S.Z. Chen ${ }^{1,3}$, T.L. Chen ${ }^{16}$,X.L. $\mathrm{Chen}^{1,2,3}$, Y. Chen ${ }^{10}$, N. Cheng ${ }^{1,3}$, Y.D. Cheng ${ }^{1,3}$, S.W. Cui ${ }^{14}$, X.H. Cui ${ }^{17}$, Y.D. Cui ${ }^{18}$, B. D'Ettorre Piazzoli ${ }^{19}$, B.Z. Dai ${ }^{20}$, H.L. Dai $^{1,3,6}$, Z.G. Dai ${ }^{7}$, Danzengluobu ${ }^{16}$, D. della Volpe ${ }^{21}$, X.J. Dong ${ }^{1,3}$, K.K. Duan ${ }^{13}$, J.H. Fan $^{11}$, Y.Z. Fan ${ }^{13}$, Z.X. Fan ${ }^{1,3}$, J. Fang ${ }^{20}$, K. Fang $^{1,3}$, C.F. Feng ${ }^{22}$, L. Feng ${ }^{13}$, S.H. Feng ${ }^{1,3}$, Y.L. Feng ${ }^{13}$, B. Gao ${ }^{1,3}$, C.D. Gao ${ }^{22}$, L.Q. Gao ${ }^{1,2,3}$, Q. Gao ${ }^{16}$, W. Gao ${ }^{22}$, M.M. Ge $^{20}$, L.S. Geng ${ }^{1,3}$, G.H. Gong ${ }^{23}$, Q.B. Gou ${ }^{1,3}$, M.H. Gu${ }^{1,3,6}$, F.L. Guo ${ }^{15}$, J.G. Guo ${ }^{1,2,3}$, X.L. Guo ${ }^{8}$, Y.Q. Guo ${ }^{1,3}$, Y.Y. Guo ${ }^{1,2,3,13}$, Y.A. $\mathrm{Han}^{24}$, H.H. He $\mathrm{H}^{1,2,3}$, H.N. He ${ }^{13}$, J.C. He ${ }^{1,2,3}$, S.L. He ${ }^{11}$, X.B. He ${ }^{18}$, Y. He ${ }^{8}$, M. Heller ${ }^{21}$, Y.K. Hor ${ }^{18}$, C. Hou ${ }^{1,3}$, H.B. Hu ${ }^{1,2,3}$, S. $\mathrm{Hu}^{9}$, S.C. $\mathrm{Hu}^{1,2,3}$, X.J. $\mathrm{Hu}^{23}$, D.H. Huang ${ }^{8}$, Q.L. Huang ${ }^{1,3}$, W.H. Huang ${ }^{22}$, X.T. Huang ${ }^{22}$, X.Y. Huang ${ }^{13}$, Z.C. Huang ${ }^{8}$, F. Ji ${ }^{1,3}$, X.L. $\mathrm{Ji}^{1,3,6}$, H.Y. Jia ${ }^{8}$, K. Jiang ${ }^{6,7}$, Z.J. Jiang ${ }^{20}$, C. Jin ${ }^{1,2,3}$, T. Ke ${ }^{1,3}$, D. Kuleshov ${ }^{25}$, K. Levochkin ${ }^{25}$, B.B. Li ${ }^{14}$, Cheng Li ${ }^{6,7}$, Cong Li ${ }^{1,3}$,

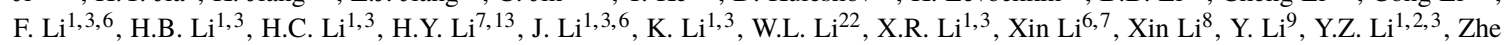

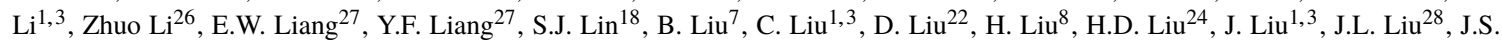

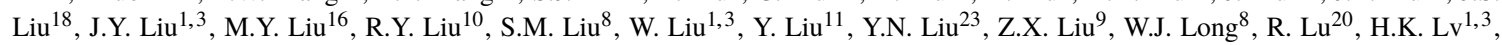

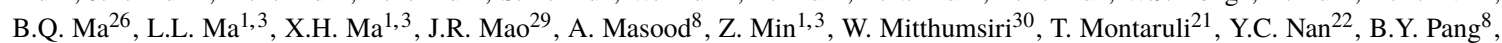
P. Pattarakijwanich ${ }^{30}$, Z.Y. Pei ${ }^{11}$, M.Y. Qi ${ }^{1,3}$, Y.Q. Qi ${ }^{14}$, B.Q. Qiao ${ }^{1,3}$, J.J. Qin ${ }^{7}$, D. Ruffolo ${ }^{30}$, V. Rulev ${ }^{25}$, A. Sáiz ${ }^{30}$, L. Shao ${ }^{14}$, O. Shchegolev $^{25,31}$, X.D. Sheng ${ }^{1,3}$, J.Y. Shi ${ }^{1,3}$, H.C. Song ${ }^{26}$, Yu.V. Stenkin ${ }^{25,31}$, V. Stepanov ${ }^{25}$, Y. Su ${ }^{32}$, Q.N. Sun ${ }^{8}$, X.N. Sun ${ }^{27}$, Z.B.

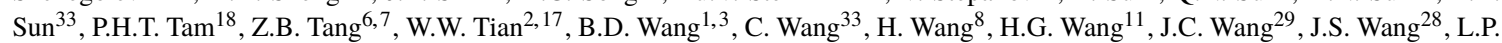
Wang $^{22}$, L.Y. Wang ${ }^{1,3}$, R.N. Wang ${ }^{8}$, W. Wang ${ }^{18}$, W. Wang ${ }^{12}$, X.G. Wang ${ }^{27}$, X.J. Wang ${ }^{1,3}$, X.Y. Wang ${ }^{10}$, Y. Wang ${ }^{8}$, Y.D. Wang ${ }^{1,3}$, Y.J. Wang $^{1,3}$, Y.P. Wang ${ }^{1,2,3}$, Z.H. Wang ${ }^{9}$, Z.X. Wang ${ }^{20}$, Zhen Wang ${ }^{28}$, Zheng Wang ${ }^{1,3,6}{ }^{3}$ D.M. Wei ${ }^{13}$, J.J. Wei ${ }^{13}$, Y.J. Wei ${ }^{1,2,3}$, T. Wen ${ }^{20}$, C.Y. $\mathrm{Wu}^{1,3}$, H.R. Wu ${ }^{1,3}$, S. Wu ${ }^{1,3}$, W.X. Wu ${ }^{8}$, X.F. Wu ${ }^{13}$, S.Q. Xi ${ }^{1,3}$, J. Xia ${ }^{7}, 13$, J.J. Xia ${ }^{8}$, G.M. Xiang ${ }^{2,15}$, D.X. Xiao ${ }^{16}$, G. Xiao ${ }^{1,3}$, H.B. Xiao ${ }^{11}$, G.G. Xin ${ }^{12}$, Y.L. Xin ${ }^{8}$, Y. Xing ${ }^{15}$, D.L. Xu ${ }^{28}$, R.X. Xu ${ }^{26}$, L. Xue ${ }^{22}$, D.H. Yann ${ }^{29}$, J.Z. Yan ${ }^{13}$, C.W. Yang ${ }^{9}$, F.F. Yang ${ }^{1,3,6}$, J.Y. Yang ${ }^{18}$, L.L. Yang ${ }^{18}$, M.J. Yang ${ }^{1,3}$, R.Z. Yang ${ }^{7}$, S.B. Yang ${ }^{20}$, Y.H. Yao ${ }^{9}$, Z.G. Yao ${ }^{1,3}$, Y.M. Ye ${ }^{23}$, L.Q. Yin ${ }^{1,3}$, N. Yin ${ }^{22}$, X.H. You $^{1,3}$, Z.Y. You ${ }^{1,2,3}$, Y.H. Yu ${ }^{22}$, Q. Yuan ${ }^{13}$, H.D. Zeng ${ }^{13}$, T.X. Zeng ${ }^{1,3,6}$, W. Zeng ${ }^{20}$, Z.K. Zeng ${ }^{1,2,3}$, M. Zha ${ }^{1,3}$, X.X. Zhai ${ }^{1,3}$, B.B. Zhang ${ }^{10}$, H.M. Zhang ${ }^{10}$, H.Y. Zhang ${ }^{22}$, J.L. Zhang ${ }^{17}$, J.W. Zhang ${ }^{9}$, L.X. Zhang ${ }^{11}$, Li Zhang ${ }^{20}$, Lu Zhang ${ }^{14}$, P.F. Zhang ${ }^{20}$, P.P. Zhang ${ }^{14}$, R. Zhang ${ }^{7,13}$, S.R. Zhang ${ }^{14}$, S.S. Zhang ${ }^{1,3}$, X. Zhang ${ }^{10}$, X.P. Zhang ${ }^{1,3}$, Y.F. Zhang ${ }^{8}$, Y.L. Zhang ${ }^{1,3}$, Yi Zhang ${ }^{1,13}$, Yong Zhang ${ }^{1,3}$, B. Zhao $^{8}$, J. Zhao ${ }^{1,3}$, L. Zhao ${ }^{6,7}$, L.Z. Zhao ${ }^{14}$, S.P. Zhao ${ }^{13,22}$, F. Zheng ${ }^{33}$, Y. Zheng ${ }^{8}$, B. Zhou ${ }^{1,3}$, H. Zhou ${ }^{28}$, J.N. Zhou ${ }^{15}$, P. Zhou ${ }^{10}$, R. Zhou $^{9}$, X.X. Zhou ${ }^{8}$, C.G. Zhu ${ }^{22}$, F.R. Zhu ${ }^{8}$, H. Zhu ${ }^{17}$, K.J. Zhu ${ }^{1,2,3,6}$ and X. Zuo ${ }^{1,3}$

${ }^{1}$ Key Laboratory of Particle Astrophyics \& Experimental Physics Division \& Computing Center, Institute of High Energy Physics, Chinese Academy of Sciences, 100049 Beijing, China.

${ }^{2}$ University of Chinese Academy of Sciences, 100049 Beijing, China.

${ }^{3}$ TIANFU Cosmic Ray Research Center, Chengdu, Sichuan, China.

${ }^{4}$ Dublin Institute for Advanced Studies, 31 Fitzwilliam Place, 2 Dublin, Ireland.

${ }^{5}$ Max-Planck-Institut for Nuclear Physics, P.O. Box 103980, 69029 Heidelberg, Germany.

${ }^{6}$ State Key Laboratory of Particle Detection and Electronics, China.

${ }^{7}$ University of Science and Technology of China, 230026 Hefei, Anhui, China.

${ }^{8}$ School of Physical Science and Technology \& School of Information Science and Technology, Southwest Jiaotong University, 610031 Chengdu, Sichuan, China.

${ }^{9}$ College of Physics, Sichuan University, 610065 Chengdu, Sichuan, China.

${ }^{10}$ School of Astronomy and Space Science, Nanjing University, 210023 Nanjing, Jiangsu, China.

${ }^{11}$ Center for Astrophysics, Guangzhou University, 510006 Guangzhou, Guangdong, China.

${ }^{12}$ School of Physics and Technology, Wuhan University, 430072 Wuhan, Hubei, China.

${ }^{13}$ Key Laboratory of Dark Matter and Space Astronomy, Purple Mountain Observatory, Chinese Academy of Sciences, 210023 Nanjing, Jiangsu, China.

${ }^{14}$ Hebei Normal University, 050024 Shijiazhuang, Hebei, China.

${ }^{15}$ Key Laboratory for Research in Galaxies and Cosmology, Shanghai Astronomical Observatory, Chinese Academy of Sciences, 200030 Shanghai, China.

${ }^{16}$ Key Laboratory of Cosmic Rays (Tibet University), Ministry of Education, 850000 Lhasa, Tibet, China.

${ }^{17}$ National Astronomical Observatories, Chinese Academy of Sciences, 100101 Beijing, China.

${ }^{18}$ School of Physics and Astronomy \& School of Physics (Guangzhou), Sun Yat-sen University, 519000 Zhuhai, Guangdong, China.

${ }^{19}$ Dipartimento di Fisica dell’Università di Napoli ‘Federico II”, Complesso Universitario di Monte Sant'Angelo, via Cinthia, 80126 Napoli, Italy.

${ }^{20}$ School of Physics and Astronomy, Yunnan University, 650091 Kunming, Yunnan, China.

${ }^{21}$ D'epartement de Physique Nucl'eaire et Corpusculaire, Facult'e de Sciences, Universit'e de Gen 'eve, 24 Quai Ernest Ansermet, 1211 Geneva, Switzerland.

${ }^{22}$ Institute of Frontier and Interdisciplinary Science, Shandong University, 266237 Qingdao, Shandong, China.

${ }^{23}$ Department of Engineering Physics, Tsinghua University, 100084 Beijing, China.

${ }^{24}$ School of Physics and Microelectronics, Zhengzhou University, 450001 Zhengzhou, Henan, China.

${ }^{25}$ Institute for Nuclear Research of Russian Academy of Sciences, 117312 Moscow, Russia.

${ }^{26}$ School of Physics, Peking University, 100871 Beijing, China. 
${ }^{27}$ School of Physical Science and Technology, Guangxi University, 530004 Nanning, Guangxi, China.

${ }^{28}$ Tsung-Dao Lee Institute \& School of Physics and Astronomy, Shanghai Jiao Tong University, 200240 Shanghai, China.

${ }^{29}$ Yunnan Observatories, Chinese Academy of Sciences, 650216 Kunming, Yunnan, China.

${ }^{30}$ Department of Physics, Faculty of Science, Mahidol University, 10400 Bangkok, Thailand.

${ }^{31}$ Moscow Institute of Physics and Technology, 141700 Moscow, Russia.

${ }^{32}$ Key Laboratory of Radio Astronomy, Purple Mountain Observatory, Chinese Academy of Sciences, 210023 Nanjing, Jiangsu, China.

${ }^{33}$ National Space Science Center, Chinese Academy of Sciences, 100190 Beijing, China. 